\title{
A Discrete Firefly Algorithm for Routing Optimization of Milk-Run
}

\author{
Xiaolei Wang ${ }^{1, a^{*}}$, Meiliang $\mathrm{He}^{2, \mathrm{~b}}$ and Hongchun Jiang ${ }^{1, \mathrm{c}}$ \\ ${ }^{1 *}$ Department of Mechanical Engineering, North China Electric Power University, Baoding, hebei, \\ China \\ ${ }^{2}$ Department of Economics and Trade, Hebei Finance University, Baoding, hebei, China \\ awxlwx1916@126.com, bpuma16@126.com, spring.jh@163.com
}

Keywords: Milk run; Route optimization; Discrete firefly algorithm

Abstract. Route planning and optimization is significant for milk run system. Therefore, taking minimizing the transportation cost as objective, a route optimization model of milk run was developed. For the discreteness of solution, a discrete firefly algorithm based on bi-layer coding was proposed. Finally, through a computational experiment, the feasibility and effectiveness of the model and algorithm were verified. Simulation results show that the algorithm has advantages in precision and convergence speed.

\section{Introduction}

Milk run, as one of the most efficient and popular model to improve logistic operations, is applied in manufacturing, food distribution, as well as military. It can reduce inventory effectively, while increasing the material flow between facilities and the usage rate of the vehicles, therefore decreasing the cost and improving the productivity. However, the vehicle routing planning (VRP) of milk run belongs to non-deterministic polynomial hard problem. With the increasing number of suppliers, the amount of available route performs exponential growth. It is complex and difficult to optimizing vehicle routing program. Therefore, many researchers have concentrated on VRP problems of milk run. Most of them focused on mathematical model and intelligent algorithm such as tabu search algorithm[1], genetic algorithm[2] and ant colony algorithm[3] to solve this problem.

As one of the biology-derived algorithms, the firefly algorithm (FA) was recently presented by Yang [4]. It was proved that FA is more efficient than genetic algorithm and particle swarm algorithms when dealing with multimodal functions[5]. It is applied in travelling salesman problems[6] and flow shop scheduling problems[7].

But adopting firefly algorithm to resolve milk run routing problem is limited. Therefore, the mathematical model of routing optimization for milk run is constructed firstly. Then, combined with the characteristic of milk run, a discrete firefly algorithm is proposed. Finally, the feasibility and validity of the proposed algorithm is verified.

\section{Route Optimization Model of Milk Run}

In this paper, the routing problem of milk run can be described as the manufacturing enterprise has a distribution center and $n$ suppliers in different cities.

Parameters:

$i$ : the $i$ th stock point or supplier; when $i=0$, it denotes this point is distribution center;

$k$ : the $k$ th route line;

$\mathrm{N}$ : the maximum number of the suppliers;

$\mathrm{M}$ : the maximum number of the route lines; 
$d_{i j}$ : the distance between supplier $i$ and supplier $j$;

$c_{i j}$ : the translation price from supplier $i$ to supplier $j$;

$q_{i}$ : the pickup amount from supplier $i$

$Q$ : the maximum load capability of the vehicles;

$c_{f}$ : the usage fixed cost of vehicle per $\mathrm{km}$.

Decision variables:

$$
\begin{gathered}
x_{i j k}=\left\{\begin{array}{l}
1, \text { the routeline " } \mathrm{k} " \text { is from point" } \mathrm{i} " \text { to point } \mathrm{j} " \\
0, \text { else }
\end{array}\right. \\
\mathrm{y}_{i k}=\left\{\begin{array}{l}
1, \text { point" } \mathrm{i} " \text { is assignedin therouteline" } \mathrm{k} " \\
0, \text { else }
\end{array}\right.
\end{gathered}
$$

Objective:

The objective is minimizing the transportation cost:

$$
\min Z=\sum_{i=0}^{N} \sum_{j=0}^{N} \sum_{k=1}^{M} c_{i j} d_{i j} x_{i j k}+\sum_{i=0}^{N} \sum_{j=0}^{N} \sum_{k=1}^{M} c_{f} d_{i j} x_{i j k}
$$

Constraints:

$$
\begin{aligned}
& \sum_{i=1}^{N} x_{0 i k}=1, \forall k=1,2, \mathrm{~L}, M \\
& \sum_{i=1}^{N} x_{i 0 k}=1, \forall k=1,2, \mathrm{~L}, M \\
& \sum_{i=1}^{N} e_{i k} y_{i k} \leq Q, k=1,2, \mathrm{~L}, M \\
& \sum_{k=1}^{M} y_{i k}=1, i=1,2, \mathrm{~L}, N \\
& \sum_{i=1}^{N} x_{i j k}=y_{i k}, j=1,2, \mathrm{~L}, N ; \forall k \\
& \sum_{j=1}^{N} x_{i j k}=y_{i k}, i=1,2, \mathrm{~L}, N ; \forall k
\end{aligned}
$$

Constraint (4) and Constraint (5) ensure that all the vehicles departure from the distribution center and go back to the distribution center after a cycle. Constraint (6) ensures that the loading amount of each vehicle cannot exceed the maximum loading capacity. Constraint (7) ensures that the parts of a supplier just can be picked up by a vehicle. Constraint (8) and Constraint (9) ensure that each point or supplier $i$ is just only assigned to one route.

In this paper the following assumptions are also made.

(1) All the vehicles in the model are identical, and they have the same maximum load capability;

(2) Manufacturing system is pull-based;

(3) The supply quantity or demand is known;

(4) Manufacturing enterprise has enough available vehicles to pickup or delivery;

(5) Each supplier corresponds to a material;

(6) The demanded amount of a supplier cannot occupy a car. If can, direct delivery mode is 
adopted;

(7) The speed and the usage fixed cost of the vehicle per $\mathrm{km}$ are constants.

\section{Firefly Algorithm}

The firefly algorithm (FA) is a population-based algorithm developed by Yang [4]. Fireflies are characterized by their flashing light and the FA is based on the following three idealized behavior of the flashing characteristics of fireflies [5]:

(1) All fireflies are unisex and are attracted to other fireflies regardless of their sex.

(2) Their attractiveness is proportional to their light intensity. Thus, for any two flashing fireflies, the less bright one will moves toward the brighter one. The attractiveness and brightness of two fireflies decrease as distance between them increases. If any two flashing fireflies have the same brightness, then they move randomly.

(3) The brightness of a firefly is determined by the objective function to be optimized.

Light intensity and attractiveness are two significant issues need to be defined in the FA.

The light intensity is expressed as follows:

$$
I(r)=I_{0} \times e^{-\gamma r^{2}}
$$

where $I_{0}$ is the original light intensity of firefly itself; $\gamma$ is a fixed light absorption coefficient; and $r$ is the distance between two fireflies.

The attractiveness of a firefly is determined by its light intensity, the attractiveness of a firefly is defined as:

$$
\beta=\beta_{0} \times e^{-\gamma r^{2}}
$$

Where $\beta_{0}$ is the attractiveness at $r=0$, is a positive constant.

The distance between any two fireflies $i$ and $j$, at position $x_{i}$ and $x_{j}$, is expressed as:

$$
r_{i j}=\left\|x_{i}-x_{j}\right\|=\sqrt{\sum_{k=1}^{k=n}\left(x_{i k}-x_{j k}\right)^{2}}
$$

The movements of fireflies consist of three terms: the current position of firefly $i$, attractiveness to another more attractive firefly, and a random walk that consists of a randomization parameter $\alpha$ and the random generated number distributed in the space $[0,1]$. The movement of firefly $i$ that is attracted to another more attractive firefly $j$ is determined by

$$
x_{i}=x_{i}+\beta_{0} e^{-\gamma_{i j}^{2}}\left(x_{j}-x_{i}\right)+\alpha\left(\text { rand }-\frac{1}{2}\right)
$$

\section{The Discrete Firefly Algorithm}

The standard firefly algorithm cannot be applied directly to solve the discrete optimization problems. In this paper, we use bi-layer coding strategy to enable that the FA can be applied to discrete routing optimization problems.

(1) Solution representation. Route and supplier's order should be included in the solution. Therefore, two vectors are used to describe this information. One is the route vector $x^{t}=\left(x_{1}^{t}\right.$, $\left.x_{2}{ }^{t} \ldots, x_{\mathrm{m}}{ }^{t}\right)$, representing the serial number of the routes. The other is the order vector $y^{t}=\left(y_{1}{ }^{t}, y_{2}{ }^{t} \ldots\right.$, $y_{\mathrm{n}}{ }^{t}$ ), representing supplier's order in the route that the supplier is assigned in. For example, the

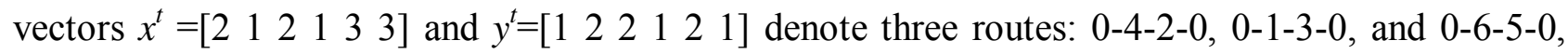
shown in Figure 1, where " 0 " represents the distribution center. 


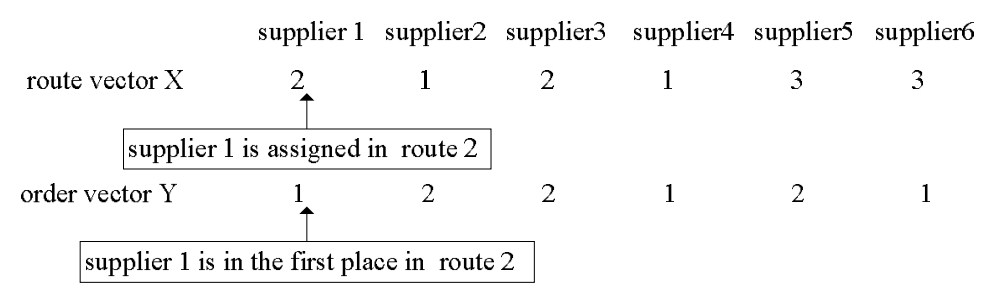

Fig. 1 Solution representation

(2) Population initialization. The initial population of DFA not only has randomness, but also ensured to satisfy the constraint of maximum loading capacity. Firstly, the suppliers are sorted randomly. Then, the suppliers are grouped from the first one. The amount of supplier provided is orderly added until reach to Q. When the added amount is greater than $\mathrm{Q}$, the supplier last added is removed, others are assigned in a route. Then, form the suppliers removed, begin a new route.

(3) Solution updation. A firefly is represented by a route vector and an order vector. The distance between two fireflies $i$ and $j$ in route vector and order vector is calculated by Eq.14 and Eq.15.

$$
\begin{aligned}
& r_{1}=\left\|x_{i}^{t}-x_{j}^{t}\right\| \\
& r_{2}=\left\|y_{i}^{t}-y_{j}^{t}\right\|
\end{aligned}
$$

The attractiveness of a firefly is determined by

$$
\begin{aligned}
& \beta_{1}=\beta_{01} \times e^{-\gamma r_{1}^{2}} \\
& \beta_{2}=\beta_{02} \times e^{-\gamma r_{2}^{2}}
\end{aligned}
$$

where $\beta_{1}$ is the attractiveness of route, $\beta_{01}$ is the maximum route attractiveness; $\gamma$ is the light absorption coefficient; $\beta_{2}$ is the attractiveness of order, $\beta_{02}$ is the maximum order attractiveness.

The less brighter firefly will be attracted and move towards the brighter one. The movement of firefly $i$ is determined by Eq.18 and Eq. 19 .

$$
\begin{aligned}
& x_{i}^{t}=x_{i}^{t}+\beta_{1}\left(x_{j}^{t}-x_{i}^{t}\right)+\alpha\left(\text { rand }-\frac{1}{2}\right) \\
& y_{i}^{t}=y_{i}^{t}+\beta_{2}\left(y_{j}^{t}-y_{i}^{t}\right)+\alpha\left(\text { rand }-\frac{1}{2}\right)
\end{aligned}
$$

(4) The light intensity of firefly. In order to calculate the fireflies' light intensity, the vector $x^{t}$ and $y^{t}$ are transformed into scheme matrix $P(\mathrm{M},(\mathrm{M}+2))$. In matrix $P$, row represents the route, column represents the supplier, and the order of the columns represents the suppliers' order in the route. The first column represents the sequence number of the route, and the last column represents the distribution center.

Based on the distance matrix $D$, price matrix $C$ and scheme matrix $P$, the light intensity of the firefly, that is the objective function value, can be got as follows.

Step 1 from $P(1,1)$ in the matrix $P$, if the $\mathrm{P}(i, 1)$ is 0 , it means there is no route exist in this row. Continue to $P(i+1,1)$. If the $P(i, 1)$ is not equal to 0 , calculating the transportation costs $T_{\text {cost }}$ according to the Eq.(20).

$$
T_{\text {cost }}=T_{\cos t}+\sum_{j=2}^{m+1} d_{(P(i, j), P(i, j+1))} c_{(P(i, j), P(i, j+1))}+\sum_{j=2}^{m+1} d_{(P(i, j), P(i, j+1))} c_{f}
$$

where when $j=1$, the $P(i, j)=P(i, j+1)$, calculate the distance from the first supplier to the distribution center; When the $P(i, j+1)=0, P(i, j+1)=P(i, j)$, calculate the distance from the last 
supplier to distribution center.

Step 2 If the $P(m, 1)=0$ or $i=m, j=m+1$, then stop; else repeat the step 1 .

\section{Computational Experiment}

This section describes the computational experiments used to evaluate the performance of the proposed algorithm. The discrete firefly algorithm was coded in MATLAB and run on a PC with an Intel Core $2.4 \mathrm{GHz}$ CPU, 4GB RAM. There are one distribution center(DC), eight suppliers $\left(\mathrm{S}_{1}, \mathrm{~S}_{2}, \ldots, \mathrm{S}_{8}\right)$ and their pickup amounts are $8,4,5,6,7,3,6,8$, respectively. The $c_{i j}$ and $c_{f}$ are constants. The distance matrix $D$ is shown in Figure 2.

$$
D=\left[\begin{array}{cccccccccc} 
& D C & S_{1} & S_{2} & S_{3} & S_{4} & S_{5} & S_{6} & S_{7} & S_{8} \\
D C & 0 & 18 & 4 & 16 & 6 & 17 & 19 & 9 & 7 \\
S_{1} & 18 & 0 & 14 & 15 & 18 & 13 & 5 & 14 & 16 \\
S_{2} & 4 & 14 & 0 & 12 & 5 & 11 & 14 & 3 & 4 \\
S_{3} & 16 & 15 & 12 & 0 & 5 & 14 & 3 & 15 & 17 \\
S_{4} & 6 & 18 & 5 & 5 & 0 & 5 & 17 & 7 & 8 \\
S_{5} & 17 & 13 & 11 & 14 & 5 & 0 & 12 & 9 & 13 \\
S_{6} & 19 & 5 & 14 & 3 & 17 & 12 & 0 & 13 & 14 \\
S_{7} & 9 & 14 & 3 & 15 & 7 & 9 & 13 & 0 & 13 \\
S_{8} & 7 & 16 & 4 & 17 & 8 & 13 & 14 & 13 & 0
\end{array}\right]
$$

Fig.2 The distance matrix

(1) Parameter Setting. The parameters of the DFA are presented in Table 1.

Table 1 The parameters of the DFA

\begin{tabular}{cccc}
\hline parameter & Its value & parameter & Its value \\
\hline the population size $(n)$ & 20 & $\gamma$ & 1 \\
$\alpha$ & 0.9 & $Q$ & 20 \\
$\beta_{01}$ & 1 & the maximum number of & 150 \\
$\beta_{02}$ & 1 & iteration(Tmax) & \\
\hline
\end{tabular}

In addition, the path random perturbation term $\mathrm{R}$ takes the value of -1 and 1 with the probability of $\alpha / 2$, takes the value of 0 with the probability of $(1-\alpha)$.

(2) Results analysis. It can be known the shorter the route, the less the cost. The optimal solution got by tabu search algorithm was 036104520870, The corresponding route scheme are 0-3-6-1-0, 0-4-5-2-0 and 0-8-7-0. Three vehicles were used and the total transportation distance was $97 \mathrm{~km}[8]$. And the optimal solution we got by the DFA is as follows: $x^{t}=\left[\begin{array}{llllllll}2 & 1 & 2 & 3 & 3 & 2 & 3 & 1\end{array}\right], y^{t}=\left[\begin{array}{lllllll}1 & 1 & 3 & 1 & 2 & 2 & 3\end{array}\right]$. The corresponding route schemes are $0-2-8-0,0-1-6-3-0,0-4-5-7-0$, three vehicles are used and the total transportation distance is $86 \mathrm{~km}$, better than the tabu search algorithm.

The iterative process of searching the optimal solution is shown in Figure 3.

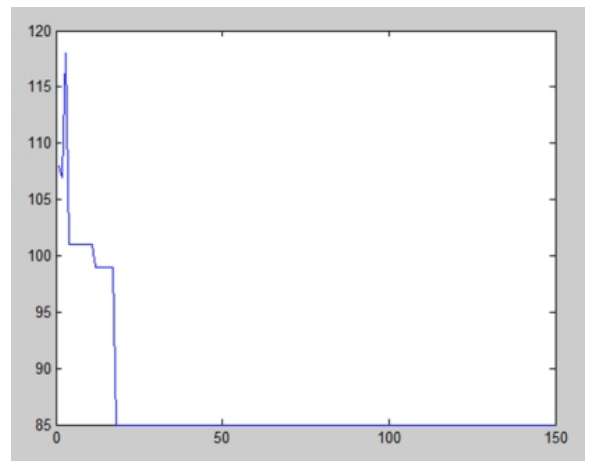

Fig.3 The iterative process of DFA 


\section{Conclusions}

In order to minimize the transportation cost of milk run, route optimization model of milk run is constructed and a discrete firefly algorithm based on bi-layer coding strategy is proposed to research the optimal solution. The firefly is expressed by route vector and order vector. The algorithm is tested with case study and the computational results show that the proposed algorithm outperforms the tabu search algorithm. The future work shall focus on the enhancing the convergence capability of the algorithm. The algorithm may also be applied to route optimization problems with determined time periods or undetermined time periods.

\section{Acknowledgements}

This research is supported by Natural Science Foundation of Hebei province (No.G2013502228), the Fundamental Research Funds for the Central Universities (No.2014 MS120) and the Science Foundation of Hebei Finance University (No.JY201506).

\section{References}

[1] J. L. Wang, Z. H. Jiang, Journal of Shanghai Jiao Tong University. 43 (2009) 1703-1709(In Chinese).

[2] L. F. Wang, P. Liu, Y. Huang, Y. C. Wang and F. S. Kong, Research on milk-run routing problem of automotive supply logistics based on genetic algorithm, International Asia Conference on Industrial Engineering and Management Innovation: Core Areas of Industrial Engineering, 2012, pp. 517-526.

[3] Z. X. Chen, B. R. Sarker, International Journal of Production Research. 52 (2014) 5086-5114.

[4] X. S. Yang, Nature-inspired metaheuristic algorithm, Bristol, U.K, 2008.

[5] X. S. Yang, Firefly algorithms for mult-imodal optimization, Proc of the 5th International Symposium on Stochastic Algorithms: Foundations and Applications, 2009, pp. 169-178.

[6] M. F. Li, J. H. Ma, Y. Y. Zhang, H.M. Zhou and J.G. Liu, Journal of Computational and Theoretical Nanoscience. 12 (2015) 1277-1281.

[7] S. Karthikeyan, P. Asokan, S. A. Nickolas, International Journal of Advanced Manufacturing Technology. 72 (2014) 1567-1579.

[8] K. Zhang, H. R. Jiang, Logistics Sci-Tech. 2 (2009) 69-72(In Chinese). 